\title{
Genetic Mapping and Molecular Characterization of a Broad-spectrum Phytophthora sojae Resistance Gene in Chinese Soybean
}

\author{
Chao Zhong ${ }^{1,+}$, Yinping $\mathrm{Li}^{1,2,+}$, Suli Sun ${ }^{1, *}$, Canxing Duan ${ }^{1}$ and Zhendong Zhu ${ }^{1, *}$ \\ 1 National Key Facility for Crop Gene Resources and Genetic Improvement, Institute of Crop Sciences, \\ Chinese Academy of Agricultural Sciences, Beijing 100081, China; tonyzhong21@163.com (C.Z.); \\ liyinpingxx@163.com (Y.L.); duancanxing@caas.cn (C.D.) \\ 2 Institute of Pomology, Chinese Academy of Agricultural Sciences, Xingcheng 125100, China \\ * Correspondence: sunsuli@caas.cn (S.S.); zhuzhendong@caas.cn (Z.Z.); Tel.: +86-10-82109609 (S.S. \& Z.Z.); \\ Fax: +86-10-82109608 (S.S. \& Z.Z.) \\ + These authors contributed equally to this work.
}

Received: 28 February 2019; Accepted: 9 April 2019; Published: 12 April 2019

\begin{abstract}
Phytophthora root rot (PRR) causes serious annual soybean yield losses worldwide. The most effective method to prevent PRR involves growing cultivars that possess genes conferring resistance to Phytophthora sojae (Rps). In this study, QTL-sequencing combined with genetic mapping was used to identify RpsX in soybean cultivar Xiu94-11 resistance to all $P$. sojae isolates tested, exhibiting broad-spectrum PRR resistance. Subsequent analysis revealed $R p s X$ was located in the 242-kb genomic region spanning the $R p s Q$ locus. However, a phylogenetic investigation indicated Xiu94-11 carrying $R p s X$ is distantly related to the cultivars containing $R p s Q$, implying $R p s X$ and $R p s Q$ have different origins. An examination of candidate genes revealed $R p s X$ and $R p s Q$ share common nonsynonymous SNP and a 144-bp insertion in the Glyma.03g027200 sequence encoding a leucine-rich repeat (LRR) region. Glyma.03g027200 was considered to be the likely candidate gene of RpsQ and $R p s X$. Sequence analyses confirmed that the 144-bp insertion caused by an unequal exchange resulted in two additional LRR-encoding fragments in the candidate gene. A marker developed based on the 144-bp insertion was used to analyze the genetic population and germplasm, and proved to be useful for identifying the $R p s X$ and $R p s Q$ alleles. This study implies that the number of LRR units in the LRR domain may be important for PRR resistance in soybean.
\end{abstract}

Keywords: next-generation sequencing; genetic mapping; RpsX; Phytophthora root rot; soybean

\section{Introduction}

Soybean [Glycine max (L.) Merr] is one of the most important economic crops, accounting for more than half of the global oilseed production [1]. Sustainable soybean cultivation is largely limited by diseases caused by diverse pathogens, including the soil-borne oomycete Phytophthora sojae, which causes Phytophthora root rot (PRR), with annual economic losses of 1-2 billion worldwide [2-6]. In China, PRR was first detected in Heilongjiang province in 1989, and it has since spread to most soybean-producing areas [7]. This disease can occur at any soybean plant developmental stage. In water-saturated soils, P. sojae zoospores can infect soybean plants throughout the growing season, causing damping-off before and after emergence, root and stem decay during the adult stage, and even death [8]. If PRR is established in the field, it is difficult to control with chemical and biological agents, ultimately resulting in considerable or complete yield losses [7]. The most effective way to control PRR currently involves growing soybean cultivars expressing Rps genes, which confer resistance to P. sojae [9]. Compared with the application of biological and chemical agents, growing PRR-resistant 
cultivars is a more environmentally friendly method for the sustainable development and production of crops.

The two types of PRR resistance have been identified in soybean, including partial resistance which is controlled by multiple genes, and complete resistance which is mediated by the single dominant $R p s$ resistance gene [9]. Soybean plants exhibiting partial resistance restrict the spread of P. sojae in plant tissues, whereas plants displaying complete resistance are immune to $P$. sojae. Furthermore, the single dominant $R p s$ genes mediating complete resistance can easily be identified and incorporated into other soybean cultivars for PRR control [9-12]. Thirty-two Rps genes have been identified to date, and they have been localized to nine soybean chromosomes [9,13-25]. However, Rps genes are race-specific and conform to the gene-for-gene hypothesis with a P. sojae avirulence gene. Several studies have revealed that the virulence of a $P$. sojae population rapidly changes, and a single race can generate various pathotypes that can overcome the resistance mediated by the Rps genes [9,26-32]. Due to the breakdown of resistance caused by the emergence of new P. sojae pathotypes, the durability of an $R p s$ gene is generally only $8-15$ years $[9,28]$. Therefore, researchers must continuously search for and identify new Rps genes.

The Rps genes have traditionally been identified and mapped to construct the corresponding mapping populations, including the $\mathrm{F}_{2: 3}$, recombinant inbred line, and near-isogenic line populations. Additionally, the mapping interval containing the Rps genes was determined by analyzing the phenotypes and polymorphic molecular markers in the segregating mapping population [18-21]. As this process necessitates the screening of many polymorphic markers between the parents and the extreme-phenotype bulks as well as the genotyping of a relatively large population, it is labor intensive and requires considerable time and resources. Recent advances in next-generation sequencing (NGS) technology have gradually decreased the costs associated with sequencing and increased the application of NGS-based methods for genomics studies related to crop improvement $[33,34]$. The sequencing, assembly, and annotation of the Williams 82 soybean reference genome have enabled the identification of increasing numbers of single nucleotide polymorphisms (SNPs) and insertions/deletions (InDels) through whole-genome resequencing and facilitated the development of key markers and the detection of Rps candidate genes [18-20,24,35-37]. Moreover, quantitative trait loci sequencing (QTL-seq) is a relatively new method that combines NGS technology with bulk segregant analysis, which is a rapid and effective approach for identifying markers linked to specific traits, to rapidly detect candidate genomic locations [38]. Identifying candidate genomic regions with sequencing data for the extreme-phenotype bulks of 20-50 selected lines has become a popular approach. The QTL-seq method has been used to map qualitative and quantitative traits in multiple plant species [39-45]. We recently identified and mapped a novel Rps gene, RpsHC18, on chromosome 3 through a combination of QTL-seq and traditional genetic mapping [24]. Thus, this is an important new method for efficiently identifying and mapping Rps genes.

China has abundant germplasm resources resistant to PRR, and some Rps genes in the corresponding cultivars/landraces have been previously reported [13,14,16,20,21,24]. Soybean cultivar Xiu94-11, which is rich in oil, was developed in Liaoning province in China. While identifying and screening PRR-resistant resources, we determined that Xiu94-11 is highly resistant to P. sojae isolates in China. Hence, the objectives of this study were to (1) characterize the inheritance of PRR resistance in Xiu94-11, (2) map the Rps gene in Xiu94-11 through QTL-seq and genetic mapping, and (3) identify the Rps candidate genes and their functional markers. 


\section{Results}

2.1. Xiu94-11 Has Broad-spectrum Resistance to Phytophthora sojae Which is Controlled by a Dominant Single Gene

The resistance to 14 P. sojae isolates with varying virulence levels was assessed for the PRR-resistant Xiu94-11 and 22 other cultivars each containing a different identified Rps gene as well as four PRR-susceptible cultivars (Zhonghuang13, Williams, Zhonghuang47, and Jikedou2) as controls. All of the soybean plants of the PRR-susceptible cultivars died after the inoculations with the P. sojae isolates. Moreover, 13 reaction types were observed among the 27 tested soybean cultivars in response to the 14 P. sojae isolates (Table 1). Specifically, Xiu94-11 was resistant to all 14 P. sojae isolates and exhibited the broadest spectrum resistance among the PRR-resistant cultivars. Thus, Xiu94-11 may contain a novel Rps gene or a unique combination of the identified Rps genes.

To analyze the genetic characteristics underlying the resistance of Xiu94-11 and further map the Rps gene(s) involved, an $\mathrm{F}_{2: 3}$ mapping population was derived from a hybridization between Zhonghuang47 and Xiu94-11. Phytophthora sojae isolates PsMC1 and PsJS2 were used to evaluate the phenotypes of $137 \mathrm{~F}_{2: 3}$ families and parental cultivars. All families exhibited consistent responses to the two P. sojae isolates. The 38 homozygous resistant families, 63 segregating families, and 36 susceptible families fit the expected 1:2:1 ratio (Table 2). These results suggested that the resistance of Xiu94-11 is controlled by a single dominant gene, which we tentatively named $R p s X$. 
Table 1. Phenotypic responses of 27 soybean cultivars to 14 Phytophthora sojae isolates.

\begin{tabular}{|c|c|c|c|c|c|c|c|c|c|c|c|c|c|c|}
\hline Cultivar (Rps gene) & PsRace1 & PsRace3 & PsRace4 & PsRace5 & PsUSAR2 & Ps41-1 & PsAH4 & PsMC1 & PsNKI & PsFJ2 & PsFJ3 & PsJS2 & Ps6497 & Ps7063 \\
\hline Harlon (Rps1a) & $S^{1}$ & $S$ & $S$ & $S$ & $\mathrm{R}$ & S & $\mathrm{R}$ & $S$ & $\mathrm{R}$ & $S$ & S & $S$ & $\mathrm{R}$ & S \\
\hline Harosoy13XX (Rps1b) & $\mathrm{R}$ & $\mathrm{R}$ & $\mathrm{R}$ & $\mathrm{R}$ & $S$ & $S$ & S & $S$ & $S$ & $S$ & $S$ & $S$ & S & $\mathrm{R}$ \\
\hline Williams79 (Rps1c) & $\mathrm{R}$ & $\mathrm{R}$ & $\mathrm{R}$ & $\mathrm{R}$ & $\mathrm{R}$ & $\mathrm{R}$ & S & $\mathrm{R}$ & $\mathrm{R}$ & $\mathrm{R}$ & $\mathrm{R}$ & $S$ & $\mathrm{R}$ & $\mathrm{R}$ \\
\hline PI103091 (Rps1d) & $\mathrm{R}$ & $\mathrm{S}$ & $\mathrm{S}$ & $\mathrm{R}$ & $\mathrm{R}$ & $\mathrm{S}$ & $\mathrm{S}$ & $S$ & $\mathrm{~S}$ & S & S & $S$ & S & S \\
\hline Williams82 (Rps1k) & $\mathrm{R}$ & $\mathrm{R}$ & $\mathrm{R}$ & $\mathrm{R}$ & $\mathrm{R}$ & $\mathrm{R}$ & $\mathrm{S}$ & $S$ & $\mathrm{R}$ & $\mathrm{R}$ & S & $S$ & $\mathrm{R}$ & $\mathrm{R}$ \\
\hline L76-988 (Rps2) & $\mathrm{R}$ & $\mathrm{R}$ & $\mathrm{R}$ & $\mathrm{R}$ & $\mathrm{S}$ & $\mathrm{S}$ & S & $S$ & $\mathrm{~S}$ & $\mathrm{~S}$ & S & $S$ & $S$ & $\mathrm{~S}$ \\
\hline L83-570 (Rps3a) & $\mathrm{R}$ & $\mathrm{R}$ & $\mathrm{R}$ & $\mathrm{R}$ & $\mathrm{R}$ & $S$ & $S$ & $S$ & $S$ & $S$ & $S$ & $S$ & $\mathrm{R}$ & S \\
\hline PRX146-36 (Rps3b) & $\mathrm{R}$ & $\mathrm{R}$ & S & $\mathrm{R}$ & $\mathrm{R}$ & $\mathrm{S}$ & $\mathrm{S}$ & $S$ & $\mathrm{~S}$ & S & S & $S$ & $\mathrm{R}$ & $\mathrm{R}$ \\
\hline PRX145-48 (Rps3c) & $\mathrm{R}$ & $\mathrm{R}$ & $\mathrm{R}$ & $\mathrm{R}$ & $\mathrm{S}$ & $S$ & S & S & $S$ & S & S & $S$ & $S$ & $\mathrm{R}$ \\
\hline L85-2352 (Rps4) & $\mathrm{R}$ & $\mathrm{R}$ & $\mathrm{R}$ & $\mathrm{R}$ & $\mathrm{R}$ & $S$ & $\mathrm{~S}$ & $S$ & $\mathrm{~S}$ & $S$ & S & $\mathrm{S}$ & $\mathrm{R}$ & S \\
\hline L85-3059 (Rps5) & $\mathrm{R}$ & $\mathrm{R}$ & $\mathrm{R}$ & $\mathrm{R}$ & $S$ & $S$ & $S$ & S & $S$ & S & $S$ & $S$ & $\mathrm{R}$ & $S$ \\
\hline Harosoy62XX (Rps6) & $\mathrm{R}$ & $\mathrm{R}$ & $\mathrm{R}$ & $\mathrm{R}$ & $\mathrm{R}$ & $\mathrm{S}$ & $\mathrm{R}$ & $S$ & $\mathrm{~S}$ & S & S & $S$ & $\mathrm{R}$ & $S$ \\
\hline Harosoy (Rps7) & $\mathrm{R}$ & $\mathrm{R}$ & $\mathrm{R}$ & $\mathrm{S}$ & $\mathrm{R}$ & $S$ & $\mathrm{~S}$ & $\mathrm{~S}$ & $\mathrm{~S}$ & $S$ & S & $\mathrm{S}$ & $\mathrm{S}$ & S \\
\hline PI399073 (Rps8) & $\mathrm{R}$ & $\mathrm{R}$ & $\mathrm{R}$ & $\mathrm{R}$ & $\mathrm{R}$ & $\mathrm{S}$ & $\mathrm{R}$ & $S$ & $\mathrm{~S}$ & S & S & S & $\mathrm{R}$ & $S$ \\
\hline Youbian30 (RpsYB30) & $\mathrm{R}$ & $\mathrm{R}$ & $\mathrm{S}$ & $\mathrm{R}$ & $\mathrm{R}$ & $\mathrm{R}$ & $S$ & $S$ & $\mathrm{R}$ & $\mathrm{R}$ & S & $S$ & $S$ & S \\
\hline Yudou25 (Rps YD25) & $\mathrm{R}$ & $\mathrm{R}$ & $\mathrm{R}$ & $\mathrm{R}$ & $\mathrm{R}$ & $\mathrm{R}$ & S & $\mathrm{R}$ & $\mathrm{R}$ & S & $\mathrm{R}$ & $S$ & $\mathrm{R}$ & $\mathrm{R}$ \\
\hline Yudou29 (RpsYD29) & $\mathrm{R}$ & $\mathrm{R}$ & $\mathrm{R}$ & $\mathrm{R}$ & $\mathrm{R}$ & $\mathrm{R}$ & S & $\mathrm{R}$ & $\mathrm{R}$ & $\mathrm{R}$ & $\mathrm{R}$ & S & $\mathrm{R}$ & $\mathrm{R}$ \\
\hline Ludou4 (Rps9) & $\mathrm{R}$ & $\mathrm{R}$ & $\mathrm{R}$ & $\mathrm{R}$ & $\mathrm{R}$ & $\mathrm{R}$ & $\mathrm{R}$ & $\mathrm{R}$ & $\mathrm{R}$ & $\mathrm{R}$ & $S$ & $\mathrm{R}$ & $\mathrm{R}$ & $\mathrm{R}$ \\
\hline Qichadou $1(R p s Q)$ & $\mathrm{R}$ & $\mathrm{R}$ & $\mathrm{R}$ & $\mathrm{R}$ & $\mathrm{R}$ & $\mathrm{R}$ & $\mathrm{R}$ & $\mathrm{R}$ & $\mathrm{R}$ & $\mathrm{R}$ & $S$ & $\mathrm{R}$ & $\mathrm{R}$ & $\mathrm{R}$ \\
\hline Wandou15 (Rps10) & $\mathrm{R}$ & $\mathrm{R}$ & $\mathrm{R}$ & $\mathrm{R}$ & $\mathrm{R}$ & $\mathrm{R}$ & $\mathrm{R}$ & $\mathrm{R}$ & $\mathrm{R}$ & S & $\mathrm{R}$ & $S$ & $\mathrm{R}$ & $\mathrm{S}$ \\
\hline Zaoshu18 (RpsZS18) & $\mathrm{R}$ & $\mathrm{R}$ & $\mathrm{R}$ & $\mathrm{R}$ & $\mathrm{R}$ & $\mathrm{R}$ & $\mathrm{S}$ & $\mathrm{S}$ & $\mathrm{R}$ & $\mathrm{R}$ & $\mathrm{R}$ & S & $\mathrm{R}$ & S \\
\hline Huachun18 (RpsHC18) & $\mathrm{R}$ & $\mathrm{R}$ & $\mathrm{R}$ & $\mathrm{R}$ & $\mathrm{R}$ & $\mathrm{R}$ & $\mathrm{R}$ & $\mathrm{R}$ & $\mathrm{R}$ & $\mathrm{R}$ & $\mathrm{R}$ & $\mathrm{R}$ & $\mathrm{R}$ & S \\
\hline Xiu94-11 (RpsX) & $\mathrm{R}$ & $\mathrm{R}$ & $\mathrm{R}$ & $\mathrm{R}$ & $\mathrm{R}$ & $\mathrm{R}$ & $\mathrm{R}$ & $\mathrm{R}$ & $\mathrm{R}$ & $\mathrm{R}$ & $\mathrm{R}$ & $\mathrm{R}$ & $\mathrm{R}$ & $\mathrm{R}$ \\
\hline Zhonghuang13 & $S$ & $\mathrm{~S}$ & S & $\mathrm{S}$ & $S$ & $\mathrm{~S}$ & $\mathrm{~S}$ & $S$ & $\mathrm{~S}$ & S & S & S & $S$ & $\mathrm{~S}$ \\
\hline Williams (rps) & $S$ & $S$ & $S$ & $S$ & $S$ & $S$ & $S$ & $S$ & $S$ & S & $S$ & $S$ & $S$ & S \\
\hline Jikedou 2 & $S$ & $S$ & S & $S$ & $\mathrm{~S}$ & $\mathrm{~S}$ & $S$ & $S$ & $\mathrm{~S}$ & $S$ & $S$ & $S$ & $\mathrm{~S}$ & S \\
\hline Zhonghuang 47 & $S$ & $S$ & S & $S$ & $S$ & $S$ & S & $S$ & $S$ & $S$ & $S$ & $S$ & $S$ & S \\
\hline
\end{tabular}

${ }^{1}$ R: resistant; S: susceptible. 
Table 2. Genetic segregation in response to Phytophthora sojae isolates PsMC1 and PsJS2 in $137 \mathrm{~F}_{2: 3}$ families derived from a cross between soybean cultivars Zhonghuang47 and Xiu94-11.

\begin{tabular}{|c|c|c|c|c|c|c|c|c|}
\hline \multirow{2}{*}{ Parent and the Cross } & \multirow{2}{*}{ Generation } & \multirow{2}{*}{ Total Plants } & \multicolumn{3}{|c|}{$\begin{array}{c}\text { Observed } \\
\text { Number }\end{array}$} & \multicolumn{3}{|c|}{$\begin{array}{l}\text { Except Ratio } \\
\text { and Goodness of Fit }\end{array}$} \\
\hline & & & $\mathbf{R}^{1}$ & Rs & $\mathrm{S}$ & R:Rs:S & $x^{2}$ & $P$ \\
\hline Xiu94-11 & P1 & 15 & 15 & - & - & & & \\
\hline Zhonghuang47 & P2 & 15 & - & - & 15 & & & \\
\hline Zhonghuang47 × Xiu94-11 & $\mathrm{F}_{2: 3}$ & 137 & 38 & 63 & 36 & 1:2:1 & 0.93 & 0.62 \\
\hline
\end{tabular}

\subsection{The Resistance Gene RpsX in Xiu94-11 is Located on Soybean Chromosome 3}

Genomic DNA samples of Xiu94-11, Zhonghuang47, and PRR-resistant and -susceptible bulks underwent whole-genome resequencing based on an Illumina system. The PRR-resistant parent, Xiu94-11, generated $24.4 \mathrm{~Gb}$ clean reads, with an average depth of $22 \times$ and $99.33 \% 5 \times$ genome coverage, whereas the susceptible parent, Zhonghuang47, produced $24.3 \mathrm{~Gb}$ clean reads, with an average depth of $21 \times$ and $99.16 \% 5 \times$ genome coverage (Table S1). The PRR-resistant bulk (R30) consisting of an equal amount of DNA from 30 homozygous resistant families produced $61.5 \mathrm{~Gb}$ clean reads, with an average depth of $61 \times$ and $99.10 \% 5 \times$ genome coverage. The PRR-susceptible bulk (S30) representing 30 homozygous susceptible families produced $65.2 \mathrm{~Gb}$ clean reads, with an average depth of $65 \times$ and $99.20 \% 5 \times$ genome coverage (Table S1).

The QTL-seq approach was used to identify the genomic region containing RpsX [38]. A total of 1,159,236 high-quality SNPs were obtained, and the delta SNP index of each SNP was calculated. The distribution of the delta SNP index (R30-S30) on 20 chromosomes (Figure S1) at the 99\% confidence level revealed a contiguous region exceeding the threshold in the 1.05-3.55 Mb genomic region of chromosome 3 (Figure 1). Accordingly, this region represented the only candidate region for RpsX.

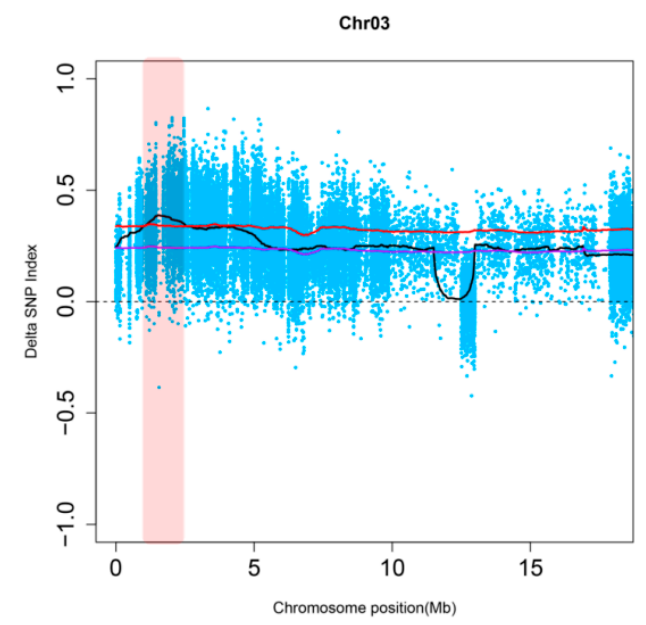

Figure 1. Delta Single nucleotide polymorphisms (SNP) index of chromosome 3. The blue dots represent the delta SNP index corresponding to an SNP obtained by filtering the two bulks. The black, purple, and red lines respectively represent the average value of the delta SNP index as well as the $95 \%$ and $99 \%$ confidence level thresholds in the corresponding window calculated by the sliding window method. The distribution of the delta SNP index at the $99 \%$ confidence level revealed only one contiguous region exceeding the threshold in the 1.05-3.55 Mb genomic region (red) of chromosome 3. The window is $1 \mathrm{Mb}$, with $10-\mathrm{kb}$ slides.

\subsection{RpsX Was Finely Mapped to the $242 \mathrm{~kb}$ Region on Chromosome 3}

To validate the accuracy of the RpsX candidate region identified by QTL-seq and further limit the $R p s X$ genomic interval, a genetic mapping approach was used to analyze all $137 \mathrm{~F}_{2: 3}$ 
families. The published simple sequence repeat (SSR) markers in the candidate region and the InDel markers developed based on the InDels identified via whole-genome resequencing were used for screening the polymorphism between the parental cultivars and clarifying the genotypes of the populations [24,25,37]. Among 150 SSR markers, seven identified polymorphisms between Xiu94-11 and Zhonghuang 47 and were closely linked to RpsX (Figure 2A). Additionally, 20 InDels between Xiu94-11 and Zhonghuang47 were developed as PCR-based markers, four of which were associated with polymorphisms and were tightly linked to RpsX (Figure 2A). Moreover, RpsX was mapped between the InDel marker InDelxz6 and the SSR marker BARCSOYSSR_03_0175, with genetic distances of 0.4 and $0.7 \mathrm{cM}$, respectively, and co-segregated with three SSR markers (BARCSOYSSR_03_0161, BARCSOYSSR_03_0165, and BARCSOYSSR_03_0167). On the basis of the physical position of each marker on chromosome 3, the genomic region comprising RpsX was localized to a 242-kb region (2,910,913-3,153,254 bp) (Figure 2B), which spans the RpsQ genomic region (Figure 2C) [20]. Polymorphisms were screened and genetic linkages were analyzed with the published InDel markers (Insert144 and Insert11) that reportedly co-segregate with $R p s Q$ [20]. The resulting data indicated that the two markers also co-segregated with $R p X$. Therefore, we speculated that $R p s X$ may be an allele of $R p s Q$.

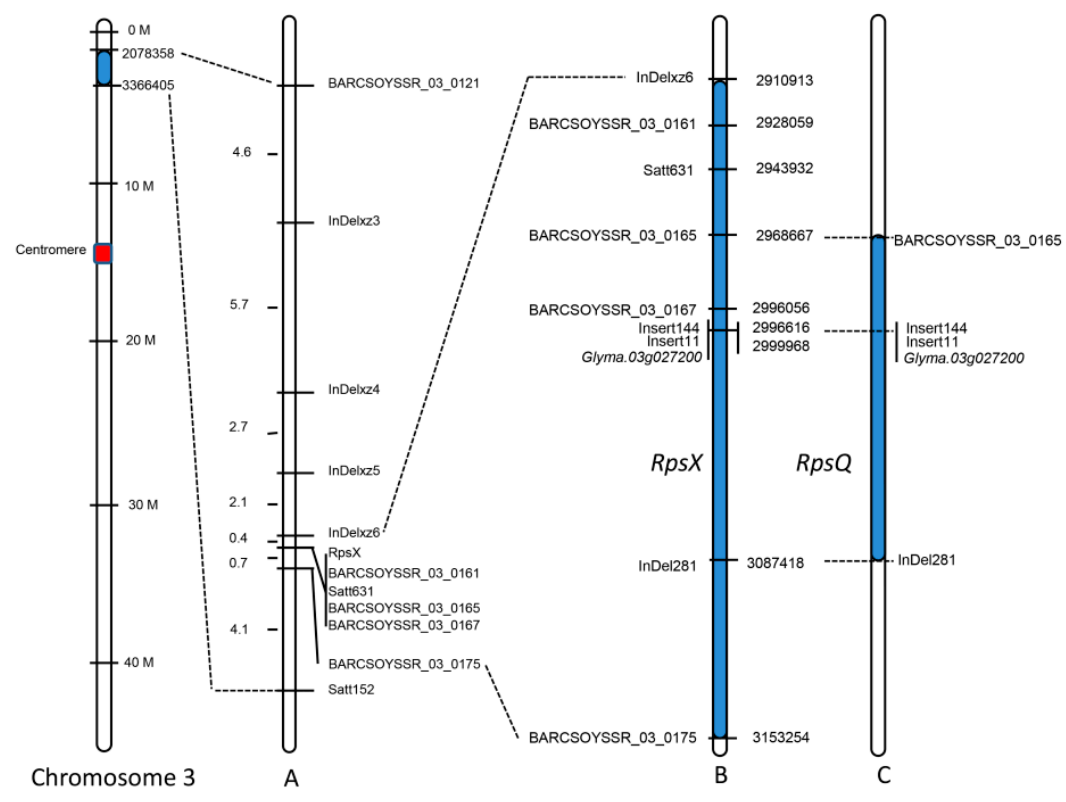

Figure 2. Genetic and physical maps of the $R p s X$ region. (A) Genetic linkage analysis of $R p s X$ based on the $\mathrm{F}_{2: 3}$ mapping population. The genetic distances $(\mathrm{cM})$ are provided on the left, and marker locations are indicated on the right. (B) Candidate physical interval of RpsX (blue region) and the physical positions of the linked and co-segregated markers of $R p s X$. (C) $R p s Q$ mapping interval and the corresponding markers [20].

\subsection{RpsX and RpsQShared the Same Candidate Gene Model}

The available information regarding the annotated soybean genome (Glyma.Wm82.a2.v1) indicates there are 24 gene models in the RpsX mapping interval (https:/www.soybase.org/). Fourteen nonsynonymous SNPs (nsSNPs) with a delta SNP index of 1 were identified distributed in eight gene models between the resistant and susceptible bulks (Figure 3). Among the eight gene models containing nsSNPs, Glyma.03g027200 containing five nsSNPs and annotated as a serine/threonine protein kinase (STK) with leucine-rich repeats (LRRs) is reportedly a type of plant resistance gene. In contrast, there are no reports suggesting the other seven gene models are related to disease resistance in plants. Therefore, Glyma.03g027200 was identified as the most likely RpsX candidate gene. Among the analyzed cultivars, the RpsX mapping interval of Xiu94-11 (RpsX) had the same nsSNP as the 
corresponding intervals of Qichadou1 (RpsQ) and the parental cultivar of Qichadou1, Ludou4 (Rps9), only for Glyma.03g027200 (Figure 3).

\begin{tabular}{|c|c|c|c|c|c|c|c|c|c|c|c|c|c|c|}
\hline Gene Model & 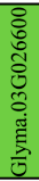 & 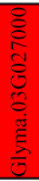 & 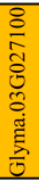 &  & 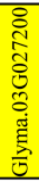 & 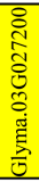 & 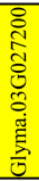 & 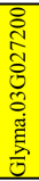 & 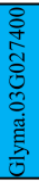 & 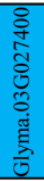 &  & 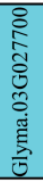 &  & 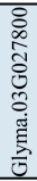 \\
\hline NsSNP Position & 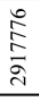 & $\begin{array}{l}\frac{a}{\infty} \\
\infty \\
\infty \\
\stackrel{\text { ते }}{0}\end{array}$ & 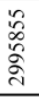 & $\begin{array}{l}\text { f } \\
\text { o } \\
\text { ¿े } \\
\text { ते }\end{array}$ & $\begin{array}{l}\frac{ \pm}{\circ} \\
\stackrel{\text { }}{\circ} \\
\stackrel{\circ}{1}\end{array}$ & $\begin{array}{l}\frac{n}{2} \\
\stackrel{2}{\circ} \\
\stackrel{\circ}{2}\end{array}$ & $\begin{array}{l}\frac{1}{2} \\
\text { مे } \\
\text { ลे }\end{array}$ & $\begin{array}{l}\overline{\hat{\delta}} \\
\text { مे } \\
\text { ㅇ }\end{array}$ & $\frac{\stackrel{\partial}{\infty}}{\partial}$ &  & $\begin{array}{l}\text { fa } \\
\text { 守 } \\
\text { ò } \\
\text { d }\end{array}$ & 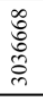 & $\begin{array}{l}0 \\
\infty \\
\infty \\
\infty \\
\infty \\
\infty\end{array}$ & 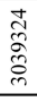 \\
\hline Willimas82(Rps $1 k)$ & $\mathrm{C}$ & $\mathrm{C}$ & $\mathrm{C}$ & $\mathrm{C}$ & $\mathrm{C}$ & $\mathrm{T}$ & $\mathrm{C}$ & A & $\mathrm{G}$ & $\mathrm{C}$ & $\mathrm{G}$ & $\mathrm{G}$ & $\mathrm{T}$ & $\mathrm{C}$ \\
\hline Williams & $\mathrm{C}$ & $\mathrm{C}$ & $\mathrm{T}$ & $\mathrm{T}$ & $\mathrm{C}$ & $\mathrm{T}$ & $\mathrm{C}$ & $\mathrm{A}$ & $\mathrm{T}$ & $\mathrm{T}$ & $\mathrm{G}$ & A & $\mathrm{C}$ & $\mathrm{T}$ \\
\hline Harlon (RpsIa) & $\mathrm{C}$ & $\mathrm{C}$ & $\mathrm{T}$ & $\mathrm{C}$ & $\mathrm{C}$ & $\mathrm{T}$ & $\mathrm{C}$ & $\mathrm{A}$ & $\mathrm{G}$ & $\mathrm{C}$ & $\mathrm{G}$ & $\mathrm{G}$ & $\mathrm{T}$ & $\mathrm{C}$ \\
\hline Harosoy13XX (Rpslb) & $\mathrm{T}$ & $\mathrm{C}$ & $\mathrm{C}$ & $\mathrm{T}$ & $\mathrm{C}$ & $\mathrm{T}$ & $\mathrm{C}$ & $\mathrm{A}$ & $\mathrm{T}$ & $\mathrm{T}$ & $\mathrm{G}$ & $\mathrm{G}$ & $\mathrm{T}$ & $\mathrm{C}$ \\
\hline Williams79 (Rpslc) & $\mathrm{C}$ & $\mathrm{C}$ & $\mathrm{C}$ & $\mathrm{C}$ & $\mathrm{C}$ & $\mathrm{T}$ & $\mathrm{C}$ & $\mathrm{A}$ & $\mathrm{G}$ & $\mathrm{C}$ & $\mathrm{G}$ & $\mathrm{G}$ & $\mathrm{T}$ & $\mathrm{C}$ \\
\hline PI103091 (Rpsld) & $\mathrm{C}$ & $\mathrm{C}$ & - & $\mathrm{T}$ & $\mathrm{C}$ & $\mathrm{T}$ & $\mathrm{C}$ & A & $\mathrm{T}$ & $\mathrm{T}$ & $\mathrm{G}$ & $\mathrm{G}$ & $\mathrm{C}$ & $\mathrm{C}$ \\
\hline Harosoy (Rps7) & $\mathrm{T}$ & A & $\mathrm{C}$ & $\mathrm{C}$ & $\mathrm{C}$ & $\mathrm{T}$ & $\mathrm{C}$ & $\mathrm{T}$ & $\mathrm{G}$ & $\mathrm{T}$ & $\mathrm{G}$ & $\mathrm{G}$ & $\mathrm{C}$ & $\mathrm{C}$ \\
\hline Qihuangl & $\mathrm{T}$ & $\mathrm{C}$ & $\mathrm{C}$ & $\mathrm{T}$ & $\mathrm{C}$ & $\mathrm{T}$ & $\mathrm{C}$ & $\mathrm{A}$ & $\mathrm{T}$ & $\mathrm{T}$ & $\mathrm{G}$ & $\mathrm{G}$ & $\mathrm{T}$ & $\mathrm{C}$ \\
\hline Dahuapi & $\mathrm{T}$ & $\mathrm{C}$ & $\mathrm{C}$ & $\mathrm{T}$ & $\mathrm{C}$ & $\mathrm{T}$ & $\mathrm{C}$ & A & $\mathrm{T}$ & $\mathrm{T}$ & $\mathrm{G}$ & $\mathrm{G}$ & $\mathrm{T}$ & $\mathrm{C}$ \\
\hline Jikedou2 & $\mathrm{C}$ & $\mathrm{C}$ & $\mathrm{C}$ & $\mathrm{C}$ & $\mathrm{C}$ & $\mathrm{T}$ & $\mathrm{C}$ & $\mathrm{A}$ & $\mathrm{T}$ & $\mathrm{T}$ & $\mathrm{G}$ & $\mathrm{G}$ & $\mathrm{C}$ & $\mathrm{C}$ \\
\hline Kaohsiungl & $\mathrm{T}$ & $\mathrm{C}$ & $\mathrm{C}$ & $\mathrm{T}$ & $\mathrm{C}$ & $\mathrm{T}$ & $\mathrm{T}$ & $\mathrm{A}$ & $\mathrm{T}$ & $\mathrm{T}$ & $\mathrm{G}$ & $\mathrm{G}$ & $\mathrm{T}$ & $\mathrm{C}$ \\
\hline Kexin3 & $\mathrm{C}$ & $\mathrm{C}$ & $\mathrm{C}$ & $\mathrm{C}$ & $\mathrm{C}$ & $\mathrm{T}$ & $\mathrm{C}$ & $\mathrm{A}$ & $\mathrm{T}$ & $\mathrm{T}$ & $\mathrm{G}$ & $\mathrm{G}$ & $\mathrm{C}$ & $\mathrm{C}$ \\
\hline Kexin4 & $\mathrm{C}$ & $\mathrm{C}$ & $\mathrm{T}$ & $\mathrm{T}$ & $\mathrm{C}$ & $\mathrm{T}$ & $\mathrm{C}$ & $\mathrm{T}$ & $\mathrm{G}$ & $\mathrm{C}$ & $\mathrm{G}$ & $\mathrm{A}$ & $\mathrm{C}$ & $\mathrm{T}$ \\
\hline Kexin5 & $\mathrm{T}$ & $\mathrm{C}$ & $\mathrm{T}$ & $\mathrm{T}$ & $\mathrm{C}$ & $\mathrm{T}$ & $\mathrm{C}$ & $\mathrm{T}$ & $\mathrm{G}$ & $\mathrm{C}$ & $\mathrm{G}$ & A & $\mathrm{C}$ & $\mathrm{T}$ \\
\hline Kexin6 & $\mathrm{C}$ & $\mathrm{C}$ & $\mathrm{C}$ & $\mathrm{C}$ & $\mathrm{C}$ & $\mathrm{T}$ & $\mathrm{C}$ & $\mathrm{A}$ & $\mathrm{T}$ & $\mathrm{T}$ & $\mathrm{G}$ & $\mathrm{G}$ & $\mathrm{C}$ & $\mathrm{C}$ \\
\hline Kexin7 & $\mathrm{C}$ & $\mathrm{C}$ & $\mathrm{C}$ & $\mathrm{C}$ & $\mathrm{C}$ & $\mathrm{T}$ & $\mathrm{C}$ & A & $\mathrm{T}$ & $\mathrm{T}$ & $\mathrm{G}$ & $\mathrm{G}$ & $\mathrm{C}$ & $\mathrm{C}$ \\
\hline Ludoul0 & $\mathrm{T}$ & $\mathrm{C}$ & $\mathrm{C}$ & $\mathrm{C}$ & $\mathrm{C}$ & $\mathrm{T}$ & $\mathrm{C}$ & A & $\mathrm{G}$ & $\mathrm{C}$ & $\mathrm{G}$ & $\mathrm{G}$ & $\mathrm{T}$ & $\mathrm{C}$ \\
\hline Ludou8 & $\mathrm{T}$ & $\mathrm{C}$ & $\mathrm{T}$ & $\mathrm{C}$ & $\mathrm{C}$ & $\mathrm{T}$ & $\mathrm{C}$ & $\mathrm{A}$ & $\mathrm{G}$ & $\mathrm{C}$ & $\mathrm{G}$ & $\mathrm{G}$ & $\mathrm{T}$ & $\mathrm{C}$ \\
\hline Maoyandou & $\mathrm{T}$ & C & $\mathrm{T}$ & $\mathrm{T}$ & $\mathrm{C}$ & $\mathrm{T}$ & $\mathrm{C}$ & $\mathrm{T}$ & $\mathrm{G}$ & C & $\mathrm{G}$ & $\mathrm{A}$ & $\mathrm{C}$ & $\mathrm{T}$ \\
\hline Meng8206 & $\mathrm{T}$ & $\mathrm{A}$ & $\mathrm{C}$ & $\mathrm{C}$ & $\mathrm{C}$ & $\mathrm{T}$ & $\mathrm{C}$ & $\mathrm{T}$ & $\mathrm{G}$ & $\mathrm{T}$ & $\mathrm{G}$ & $\mathrm{G}$ & $\mathrm{C}$ & $\mathrm{C}$ \\
\hline \begin{tabular}{|l|} 
Pingdinghuang \\
\end{tabular} & $\mathrm{T}$ & $\mathrm{C}$ & $\mathrm{C}$ & $\mathrm{C}$ & $\mathrm{C}$ & $\mathrm{T}$ & $\mathrm{C}$ & $\mathrm{A}$ & $\mathrm{G}$ & $\mathrm{C}$ & $\mathrm{G}$ & $\mathrm{G}$ & $\mathrm{T}$ & $\mathrm{C}$ \\
\hline Tianedan & $\mathrm{C}$ & $\mathrm{C}$ & $\mathrm{C}$ & $\mathrm{T}$ & $\mathrm{C}$ & $\mathrm{T}$ & $\mathrm{T}$ & $\mathrm{A}$ & $\mathrm{T}$ & $\mathrm{T}$ & $\mathrm{G}$ & $\mathrm{G}$ & $\mathrm{T}$ & $\mathrm{C}$ \\
\hline Tiejiaohuang & $\mathrm{C}$ & $\mathrm{C}$ & $\mathrm{C}$ & $\mathrm{C}$ & $\mathrm{C}$ & $\mathrm{T}$ & $\mathrm{C}$ & $\mathrm{T}$ & $\mathrm{T}$ & $\mathrm{T}$ & A & $\mathrm{G}$ & $\mathrm{C}$ & $\mathrm{C}$ \\
\hline WDD00742 & $\mathrm{T}$ & $\mathrm{C}$ & $\mathrm{C}$ & $\mathrm{T}$ & $\mathrm{C}$ & $\mathrm{T}$ & $\mathrm{C}$ & A & $\mathrm{T}$ & $\mathrm{T}$ & $\mathrm{G}$ & $\mathrm{G}$ & $\mathrm{T}$ & C \\
\hline WDD00753 & C & $\mathrm{C}$ & $\mathrm{C}$ & $\mathrm{C}$ & $\mathrm{C}$ & $\mathrm{T}$ & $\mathrm{C}$ & $\mathrm{A}$ & $\mathrm{T}$ & $\mathrm{T}$ & $\mathrm{G}$ & $\mathrm{G}$ & $\mathrm{C}$ & $\mathrm{C}$ \\
\hline Yudou2 & $\mathrm{T}$ & $\mathrm{C}$ & $\mathrm{T}$ & $\mathrm{T}$ & $\mathrm{C}$ & $\mathrm{T}$ & $\mathrm{C}$ & $\mathrm{T}$ & $\mathrm{G}$ & $\mathrm{C}$ & $\mathrm{G}$ & A & $\mathrm{C}$ & $\mathrm{T}$ \\
\hline Yudou21 & $\mathrm{T}$ & $\mathrm{C}$ & $\mathrm{T}$ & $\mathrm{C}$ & $\mathrm{C}$ & $\mathrm{T}$ & $\mathrm{C}$ & A & $\mathrm{G}$ & $\mathrm{C}$ & $\mathrm{G}$ & $\mathrm{G}$ & $\mathrm{T}$ & $\mathrm{C}$ \\
\hline Yudou22 & $\mathrm{C}$ & $\mathrm{C}$ & $\mathrm{C}$ & $\mathrm{C}$ & $\mathrm{C}$ & $\mathrm{T}$ & $\mathrm{C}$ & $\mathrm{A}$ & $\mathrm{G}$ & $\mathrm{C}$ & - & $\mathrm{G}$ & $\mathrm{T}$ & $\mathrm{C}$ \\
\hline Yudou27 & $\mathrm{C}$ & $\mathrm{C}$ & - & $\mathrm{T}$ & $\mathrm{C}$ & $\mathrm{T}$ & $\mathrm{C}$ & $\mathrm{A}$ & $\mathrm{T}$ & $\mathrm{T}$ & $\mathrm{G}$ & $\mathrm{G}$ & $\mathrm{C}$ & $\mathrm{C}$ \\
\hline Yuejin4 & $\mathrm{T}$ & $\mathrm{C}$ & $\mathrm{C}$ & $\mathrm{C}$ & $\mathrm{C}$ & $\mathrm{T}$ & $\mathrm{C}$ & $\mathrm{A}$ & $\mathrm{G}$ & $\mathrm{C}$ & $\mathrm{G}$ & $\mathrm{G}$ & $\mathrm{T}$ & $\mathrm{C}$ \\
\hline Zheng135 & $\mathrm{T}$ & $\mathrm{C}$ & $\mathrm{C}$ & $\mathrm{C}$ & $\mathrm{C}$ & $\mathrm{T}$ & $\mathrm{C}$ & $\mathrm{A}$ & $\mathrm{G}$ & $\mathrm{C}$ & $\mathrm{G}$ & $\mathrm{G}$ & $\mathrm{T}$ & $\mathrm{C}$ \\
\hline Zheng77249 & $\mathrm{T}$ & $\mathrm{C}$ & $\mathrm{C}$ & $\mathrm{T}$ & $\mathrm{C}$ & $\mathrm{T}$ & $\mathrm{C}$ & $\mathrm{A}$ & $\mathrm{T}$ & $\mathrm{T}$ & $\mathrm{G}$ & $\mathrm{G}$ & $\mathrm{T}$ & $\mathrm{C}$ \\
\hline Zheng90007 & $\mathrm{T}$ & $\mathrm{C}$ & $\mathrm{C}$ & $\mathrm{T}$ & $\mathrm{C}$ & $\mathrm{T}$ & $\mathrm{C}$ & $\mathrm{A}$ & $\mathrm{T}$ & $\mathrm{T}$ & $\mathrm{G}$ & $\mathrm{G}$ & $\mathrm{T}$ & $\mathrm{C}$ \\
\hline Zheng92116 & $\mathrm{C}$ & $\mathrm{C}$ & - & $\mathrm{T}$ & $\mathrm{C}$ & $\mathrm{T}$ & $\mathrm{C}$ & A & $\mathrm{T}$ & $\mathrm{T}$ & $\mathrm{G}$ & $\mathrm{G}$ & $\mathrm{C}$ & $\mathrm{C}$ \\
\hline Zheng97196 & $\mathrm{C}$ & $\mathrm{C}$ & - & $\mathrm{T}$ & $\mathrm{C}$ & $\mathrm{T}$ & $\mathrm{C}$ & $\mathrm{A}$ & $\mathrm{T}$ & $\mathrm{T}$ & $\mathrm{G}$ & $\mathrm{G}$ & $\mathrm{C}$ & $\mathrm{C}$ \\
\hline Zhongdou28 & $\mathrm{C}$ & $\mathrm{C}$ & $\mathrm{C}$ & $\mathrm{C}$ & $\mathrm{C}$ & $\mathrm{T}$ & $\mathrm{C}$ & $\mathrm{A}$ & $\mathrm{T}$ & $\mathrm{T}$ & $\mathrm{G}$ & $\mathrm{G}$ & C & $\mathrm{C}$ \\
\hline Zhonghuang10 & $\mathrm{T}$ & $\mathrm{C}$ & $\mathrm{C}$ & $\mathrm{C}$ & $\mathrm{C}$ & $\mathrm{T}$ & $\mathrm{C}$ & A & $\mathrm{G}$ & $\mathrm{C}$ & $\mathrm{G}$ & $\mathrm{G}$ & $\mathrm{T}$ & $\mathrm{C}$ \\
\hline Zhonghuang23 & $\mathrm{T}$ & $\mathrm{C}$ & $\mathrm{C}$ & $\mathrm{T}$ & $\mathrm{C}$ & $\mathrm{T}$ & $\mathrm{C}$ & $\mathrm{A}$ & $\mathrm{T}$ & $\mathrm{T}$ & $\mathrm{G}$ & $\mathrm{G}$ & $\mathrm{T}$ & $\mathrm{C}$ \\
\hline Zhonghuang47 & $\mathrm{C}$ & $\mathrm{A}$ & $\mathrm{C}$ & $\mathrm{C}$ & $\mathrm{C}$ & $\mathrm{T}$ & $\mathrm{G}$ & $\mathrm{A}$ & $\mathrm{T}$ & $\mathrm{T}$ & $\mathrm{A}$ & $\mathrm{G}$ & $\mathrm{T}$ & $\mathrm{C}$ \\
\hline Zhongpin661 & $\mathrm{C}$ & $\mathrm{C}$ & $\mathrm{C}$ & $\mathrm{C}$ & $\mathrm{C}$ & $\mathrm{T}$ & $\mathrm{C}$ & A & $\mathrm{T}$ & $\mathrm{T}$ & $\mathrm{G}$ & $\mathrm{G}$ & $\mathrm{C}$ & $\mathrm{C}$ \\
\hline Zhongpin662 & $\mathrm{T}$ & $\mathrm{C}$ & $\mathrm{C}$ & $\mathrm{T}$ & $\mathrm{C}$ & $\mathrm{T}$ & $\mathrm{C}$ & $\mathrm{A}$ & $\mathrm{T}$ & $\mathrm{T}$ & $\mathrm{G}$ & $\mathrm{G}$ & $\mathrm{T}$ & $\mathrm{C}$ \\
\hline Zhongpin95-5117 & $\mathrm{C}$ & $\mathrm{C}$ & $\mathrm{C}$ & $\mathrm{C}$ & $\mathrm{C}$ & $\mathrm{T}$ & $\mathrm{C}$ & A & $\mathrm{T}$ & $\mathrm{T}$ & $\mathrm{G}$ & $\mathrm{G}$ & $\mathrm{C}$ & $\mathrm{C}$ \\
\hline Ludou4(Rps9) & $\mathrm{T}$ & $\mathrm{C}$ & $\mathrm{T}$ & $\mathrm{T}$ & $\mathrm{T}$ & $\mathrm{A}$ & $\mathrm{T}$ & $\mathrm{T}$ & $\mathrm{G}$ & $\mathrm{C}$ & $\mathrm{G}$ & $\mathrm{A}$ & $\mathrm{C}$ & $\mathrm{T}$ \\
\hline Qichadoul(Rps $Q$ ) & $\mathrm{T}$ & $\mathrm{C}$ & $\mathrm{T}$ & $\mathrm{T}$ & $\mathrm{T}$ & $\mathrm{A}$ & $\mathrm{T}$ & $\mathrm{T}$ & $\mathrm{G}$ & $\mathrm{C}$ & $\mathrm{G}$ & $\mathrm{A}$ & $\mathrm{C}$ & $\mathrm{T}$ \\
\hline Xiu94-11 & $\mathrm{T}$ & $\mathrm{C}$ & $\mathrm{T}$ & $\mathrm{T}$ & $\mathrm{T}$ & A & $\mathrm{T}$ & $\mathrm{T}$ & $\mathrm{G}$ & $\mathrm{C}$ & $\mathrm{G}$ & $\mathrm{A}$ & $\mathrm{C}$ & $\mathrm{T}$ \\
\hline
\end{tabular}

Figure 3. Nonsynonymous SNPs located in the corresponding gene models of the RpsX mapping interval among 45 soybean genotypes. Different gene models are presented in different colors. A base in a white background is the same as that in the PRR-susceptible parent Zhonghuang47, whereas a base in a gray background is the same as that in the PRR-resistant parent Xiu94-11.

As Glyma.03g027200 was identified as an $R p s Q$ candidate gene, we examined whether Xiu94-11 and Qichadou1 are related and whether $R p s X$ and $R p s Q$ are actually the same gene. We completed a phylogenetic analysis based on the SNPs identified by NGS among 45 cultivars/landraces, some of which are related to Xiu94-11 and Qichadou1 (Table S2). A total of 48,049 SNPs on chromosome 3 identified among 45 cultivars were selected to construct a phylogenetic tree according to the neighbor-joining method. We observed that 45 soybean genotypes formed two subgroups, and Xiu94-11 belonged to a subgroup separate from that of Ludou4 and Qichadou1 (Figure 4). The cultivar most closely related to Xiu94-11 was Kaohsiung1, which was selected from Chu-tzu-dow, a landrace from Taiwan, which is not related to Qichadou1 [46]. These results implied that Xiu94-11 is not closely related to Qichadou1. 


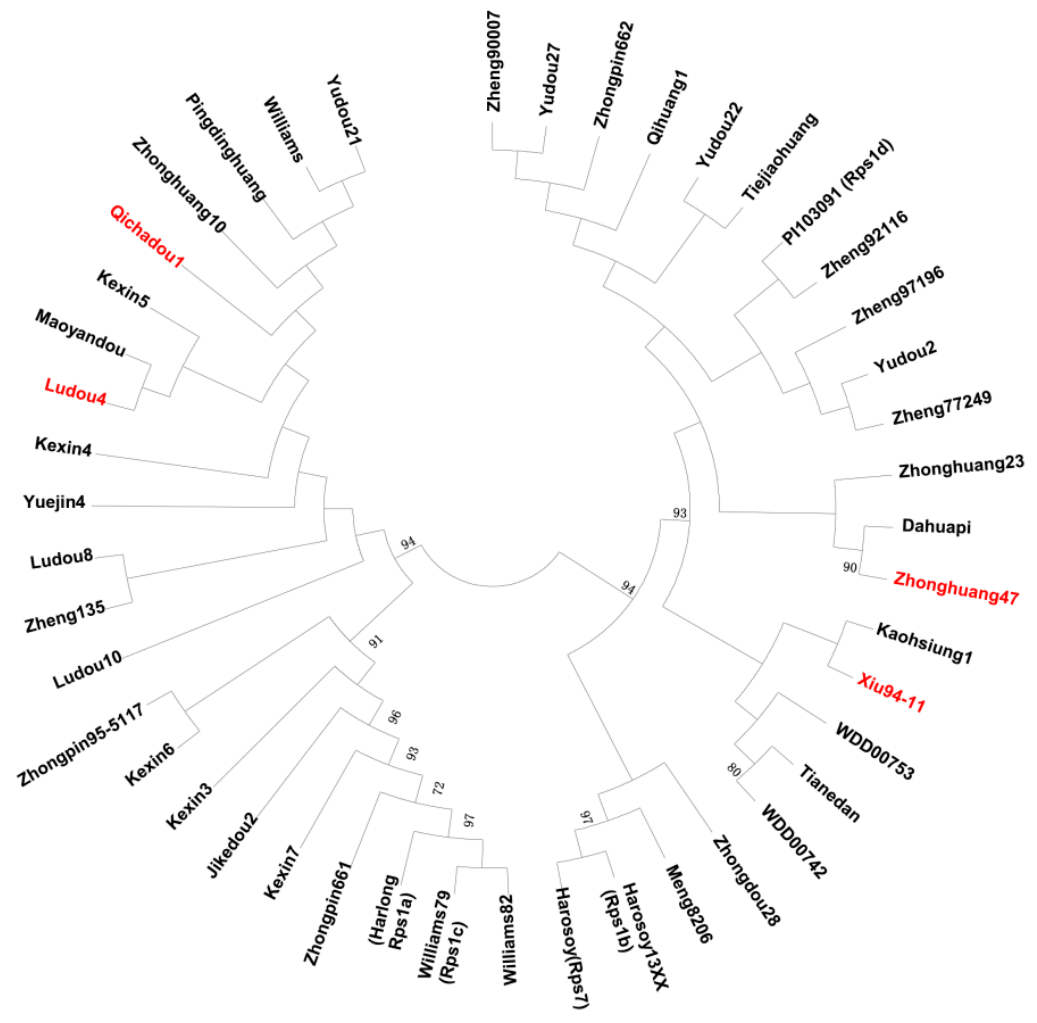

Figure 4. Phylogenetic tree constructed according to the neighbor-joining method using homozygous SNPs identified on chromosome 3 among 45 soybean genotypes, including cultivars and landraces.

\subsection{4-bp Insertion in LRR Domain is Present in Resistant Haplotype of RpsX Locus}

To further explore the candidate gene sequence and structural differences between the resistant and susceptible genotypes, the allele sequences of Glyma.03g027200 in Xiu94-11 and Zhonghuang47 were obtained with a Sanger sequencing method. All five nsSNPs identified by NGS were confirmed in Xiu94-11 and Zhonghuang47. A comparison of the obtained and the previously published RpsQ candidate gene sequences [20] revealed a 99\% sequence identity between the $R p s X$ and $R p s Q$ alleles. Additionally, we detected a $97 \%$ sequence identity between the RpsX allele and the alleles of the Williams82 reference genome sequence and the susceptible control Zhonghuang41 (Supplemental sequence S1). These results indicated that the Glyma.03g027200 sequence is relatively conserved and is highly similar between the resistant and susceptible genotypes. The deduced protein sequences based on the genomic sequences were aligned and analyzed for conserved domains. We detected two functional domains in all aligned sequences, namely the LRR and STK domains. However, the resistant and susceptible alleles differ in the sequence encoding the LRR region. Specifically, 11 LRR motifs are encoded in the $R p s X$ and $R p s Q$ alleles of the resistant genotype, whereas nine LRR motifs are encoded in the corresponding alleles of the susceptible genotypes. Like the RpsQ allele, the RpsX allele contains a 144-bp insertion, resulting in the insertion of 48 amino acid residues comprising two LRR structural units (Figure 5). The genomic sequence most similar to the 144-bp insertion is a 144-bp sequence upstream of the candidate gene, implying the insertion may have been the result of a replication of the upstream fragment (Figure 5).

Because there are currently no soybean cultivars derived from Xiu94-11, to further verify whether the detected 144-bp insertion is important for the resistance to P. sojae, soybean genotypes related to Qichadou1 based on pedigrees were analyzed regarding their reactions to 12 P. sojae isolates. Additionally, their alleles corresponding to Glyma.03g027200 were sequenced (Supplemental sequence S1). A phylogenetic tree was constructed according to the neighbor-joining method using the Glyma.03g027200 allelic sequences of 30 genotypes. Four cultivars, namely Xiu94-11 (RpsX), Qichadou1 
(RpsX), Ludou4 (Rps9), and Kexin5, were clustered in one subgroup. Moreover, the same 144-bp insertion was detected in their Glyma.03g027200 allelic sequences (Figure 6). Qichadou1 was derived from a cross between Ludou4 and Peking, whereas Kexin5 was the result of the chemical mutagenesis of Ludou4. These four cultivars all exhibited excellent resistance to 10-12 P. sojae isolates, suggesting that the 144-bp insertion in the sequence encoding the LRR region may be important for the observed resistance to $P$. sojae.

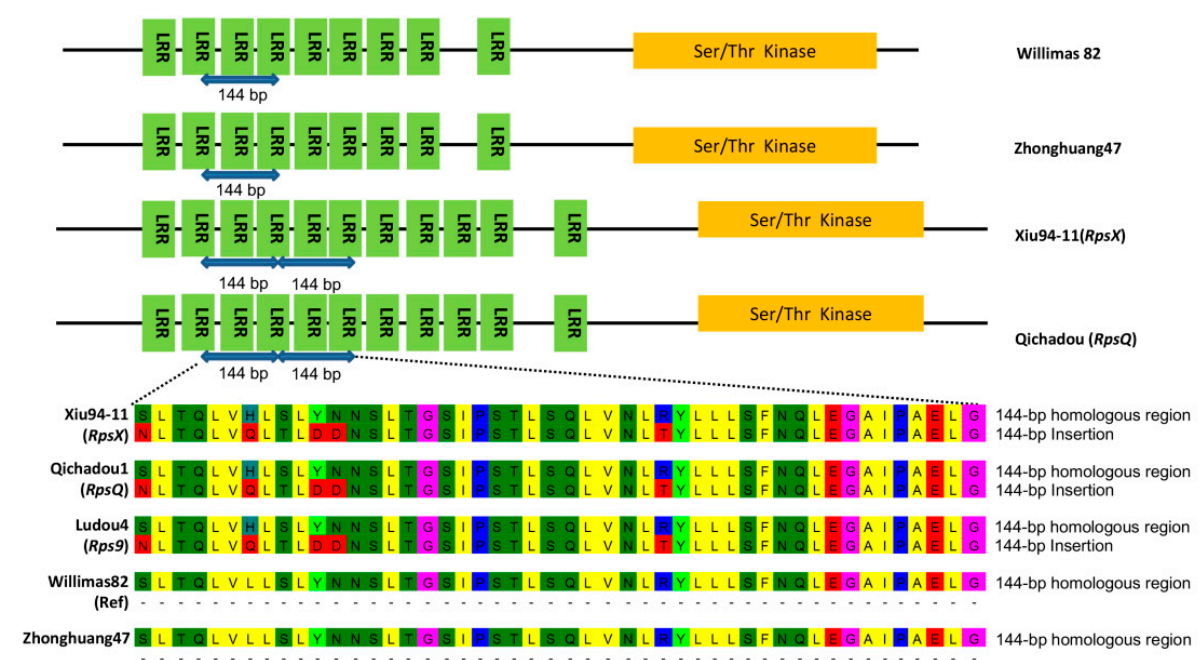

Figure 5. Analysis of conserved domains encoded by the alleles of soybean genotypes. The Rps X and $R p s Q$ alleles contain a 144-bp insertion encoding 48 amino acid residues. This insertion is most similar to a 144-bp sequence upstream of the candidate gene.

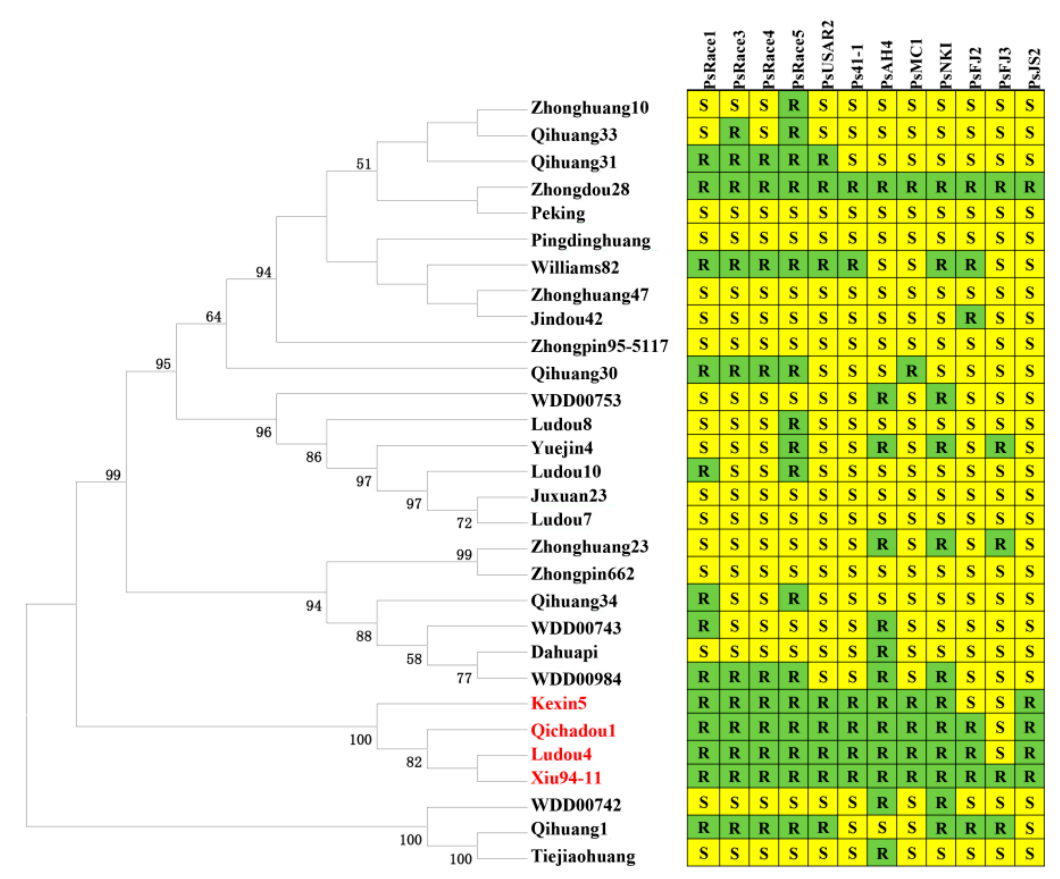

Figure 6. Phylogenetic tree constructed according to the neighbor-joining method using the allelic sequences of the candidate gene model Glyma.03g027200 in 30 soybean cultivars. The reactions of the cultivars to 12 P. sojae isolates are also presented.

\subsection{Developed Marker Insert144 Is Able to Efficiently Detect Resistant Haplotypes at RpsX Locus}

Because the 144-bp insertion is a key variant of the RpsX locus, Insert144, which is an InDel marker based on this insertion, was developed as a marker that co-segregates with $R p s Q$ and was 
used to distinguish $R p s Q$ from the $R p s 1$ alleles. In the present study, Insert144 was further validated in all cultivars carrying currently identified Rps genes as well as in the susceptible controls [20]. Only three soybean cultivars, namely Xiu94-11 (RpsX), Ludou4, and Qichadou1 (RpsQ), contained this insertion (Figure 7). The screening and identification of $R p s X$ and its alleles among 177 soybean germplasms revealed that six soybean cultivars (Ludou2, Qihuang9, Fendou78, Fendou79, Qihuang12, and Qihuang13) had the same genotype as Xiu94-11. The phenotypic responses to eight $P$. sojae isolates indicated that they were all resistant to PRR, meaning the eight cultivars are likely to contain RpsX or PRR-resistance alleles at the $R p s X$ locus. These results suggested that Insert144 can serve as a functional and diagnostic marker for RpsX and the PRR-resistance alleles during soybean breeding.

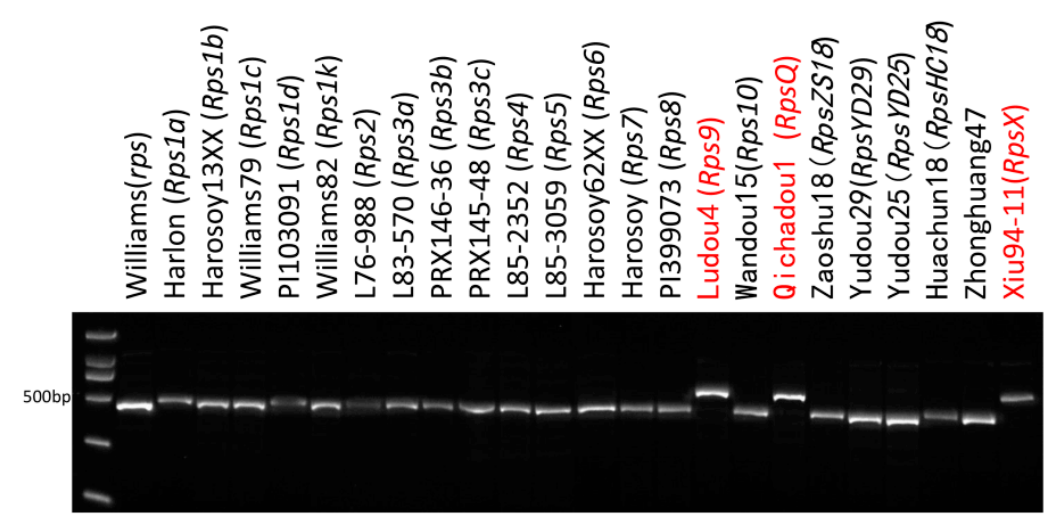

Figure 7. Molecular banding patterns for PRR-resistant cultivars containing the identified Rps genes. The cultivars were analyzed with the Insert144 marker developed based on the 144-bp insertion in Rps $X$ and $R p s Q$.

\section{Discussion}

In this study, a rapid approach combining high-throughput sequencing and traditional genetic mapping was deployed to identify a novel allele, $R p s X$, at the $R p s Q$ locus in a small $\mathrm{F}_{2: 3}$ population. The most prominent advantage of this approach is that it enables the genotyping and mapping of a resistance gene in a relatively early generation like $\mathrm{F}_{2: 3}$. Additionally, the SNPs and InDels identified based on high-throughput sequencing can be used for further fine mapping, a haplotype analysis, and the identification of candidate genes [24,25,38-45]. Phytophthora sojae virulence involves a complex mechanism and can rapidly change, enabling this pathogen to quickly overcome the resistance conferred by most $R p s$ genes, ultimately leading to severe yield losses. Thus, whole-genome resequencing represents a fast, efficient, accurate, and relatively simple method for identifying novel Rps genes [24,25].

Many of the currently known Rps genes have been mapped on the short arm of soybean chromosome 3, including some genes that have been finely mapped such as $R p s 1 k, R p s Y D 29, R p s Q$, RpsHC18, RpsWY, RpsHN, and RpsUN1 [11,20,24-27,30]. Moreover, most of these genes were mapped in the interval containing typical plant resistance genes encoding a nucleotide-binding site (NBS) and an LRR domain. Previous studies indicated that some genes encoding an NBS-LRR structure were candidate genes for these Rps genes [14,18,21,24]. The Rps1k gene, which is associated with broad-spectrum and durable resistance, has been cloned, and the tandemly arranged NBS-LRR genes $R p s 1 k-1$ and $R p s 1 k-2$ were functionally validated as responsible for conferring complete resistance to $P$. sojae $[47,48]$. Unlike the Rps genes in tandemly arranged NBS-LRR gene clusters, RpsX was mapped to an interval on the short arm of chromosome 3 lacking NBS-LRR genes. Only one plant resistance gene, Glyma.03g027200, which encodes an STK-LRR structure, was detected in the RpsX region. Additionally, Glyma.03g027200 contains an RpsX-specific nsSNP, which was identified by QTL-seq (Figure 3). These results imply that Glyma.03g027200 is the likely candidate gene for RpsX, and may represent another gene type conferring resistance to $P$. sojae. 
Interestingly, Glyma.03g027200 is also the candidate gene for $R p s Q$, which we previously identified and mapped [20]. The majority of the mapping intervals with RpsX coincided with the mapping intervals with $R p s Q$ (Figure 2B,C). A pedigree analysis revealed that Qichadou1, which contains RpsQ, was the result of a hybridization between Ludou4 and Peking, which is a PRR-susceptible cultivar [20]. In contrast, Xiu94-11 was obtained from a cross between lines 89-6 and Dandou806. Both of these lines were derived from landraces originating in northeastern China, whereas the ancestors of Qichadou1 were landraces originating in Shandong province, China, a Peking landrace, and the American cultivar Magnolia. Consequently, Xiu94-11 and Qichadou1 are not related. Moreover, our phylogenetic analysis based on the homozygous SNPs on chromosome 3 revealed a distant genetic relationship between Xiu94-11 and Qichadou1 and Ludou4. Interestingly, however, the Glyma.03g027200 allele sequences of $R p s X$ and $R p s Q$ are highly similar and carry the same SNPs and InDels. Therefore, we speculated that $R p s X$ and $R p s Q$ evolved independently via the same molecular mechanism in different ecological regions. The Glyma.03g027200 locus should be a mutation hotspot in the soybean genome, and additional PRR-resistance alleles may exist in soybean germplasm.

The Glyma.03g027200 sequence comprises an STK-LRR gene, which is another type of plant resistance gene. The STK-LRR resistance gene encodes an extracellular receptor-like protein kinase with an extracellular LRR and an intracellular STK. A few plant resistance genes have been cloned and verified to be STK-LRR genes, including rice genes conferring resistance to bacterial blight (Xa21, $X a 21 D$, and Xa3/Xa26), a wheat leaf rust resistance gene ( $L r 10)$, and an apple scab resistance candidate gene (Rvi12_Cd5) [49-53]. In the present study, the candidate gene sequences were highly similar (97\%) between the PRR-resistant genotypes Xiu94-11 (RpsX) and Qichadou1 (RpsQ) and the PRR-susceptible genotypes Zhonghuang47 and Williams82. Most of the sequence differences between the resistant and susceptible genotypes were detected in the sequence encoding the LRR domain, which plays a key role in the disease resistance mechanism of plants. The LRR domain determines the specificity of the recognition of pathogen effectors [54,55]. The LRR units containing approximately 20-30 amino acid residues consist of repeating core $x x \operatorname{Lx} \operatorname{Lx}$ motifs $(\mathrm{L}=$ leucine or other aliphatic amino acids, $\mathrm{x}=$ any amino acid) [56]. Allelic mutations due to SNPs and InDels in the sequence encoding the LRR domain may result in the generation of new $R$ genes $[57,58]$. Because of the high sequence identity between the sequences encoding the LRR units in the LRR domain, unequal crossing-over and illegitimate recombinations are prone to occur, resulting in new $R$ gene specificities due to the differences in the number of encoded LRR units [56,59,60]. Therefore, in this study, the 144-bp insertion of the RpsX and $R p s Q$ candidate genes was caused by an unequal exchange with the adjacent 144-bp fragment, resulting in a new Rps gene.

InDel marker Insert144 is a co-segregated marker developed to detect 144-bp insertions in RpsQ. Additionally, this marker can distinguish $R p s Q$ from the alleles at the $R p s 1$ locus. Thus, this marker co-segregates with and can be used to detect $R p s X$. We used this marker to analyze diverse soybean genotypes and determined that it can detect PRR-resistance alleles at the RpsX/Q locus. Therefore, Insert144 may be applied for molecular marker-assisted selection. The PRR-resistant genotypes containing the 144-bp insertion are all highly resistant to P. sojae, indicating this insertion is important for the resistance to $P$. sojae.

In conclusion, we identified a novel allele, $R p s X$, at the $R p s Q$ locus using a QTL-seq method involving high-throughput sequencing and traditional genetic mapping. A cluster analysis with homozygous SNPs on chromosome 3 and an analysis of the allelic sequences of the candidate gene confirmed that the genetic background of $R p s X$ and $R p s Q$ varies considerably, but both candidate alleles have the same 144-bp insertion in the sequence encoding the LRR domain. Therefore, changes to the LRR-encoding region play an important role in the development of novel Rps genes. The method described herein represents a rapid and efficient procedure for identifying novel $R p s$ genes and may be useful for the cloning of $R p s X$ and $R p s Q$ as well as the application of $R p s X$ and $R p s Q$ functional markers for marker-assisted selection. 


\section{Materials and Methods}

\subsection{Phenotyping for PRR Resistance}

Phytophthora root rot-resistant cultivar Xiu94-11 along with 22 cultivars with a single Rps gene and four PRR-susceptible cultivars were analyzed regarding their resistance to 14 P. sojae isolates that varied in terms of virulence. Additionally, 28 soybean cultivars and landraces related to Qichadou1 as well as Xiu94-11 and Zhonghuang47 were inoculated with 12 P. sojae isolates (Figure 6) to identify the RpsX PRR-resistance alleles. Soybean plants were grown and inoculated with $P$. sojae isolates as previously described [24,25].

A mapping population was constructed with Zhonghuang47 as the female parent and Xiu94-11 as the male parent to derive $F_{1}$ seeds. An $F_{1}$ seed was used to produce $137 \mathrm{~F}_{2: 3}$ families by self-crossing. For each family, 20-25 seeds were sown in paper cups filled with vermiculite to evaluate the responses of the resulting plants to P. sojae isolates PsMC1 and PsJS2. The plants were inoculated and their phenotypes were evaluated as previously described [24,25].

\subsection{Next-generation Sequencing and QTL-seq Analysis of Resistant and Susceptible Bulks}

On the basis of the phenotypic evaluations, 30 homozygous resistant and 30 susceptible families were respectively used to construct PRR-resistant (R30) and PRR-susceptible (S30) bulks for a subsequent NGS with an Illumina system. The generated data underwent a QTL-seq analysis [25,38]. The DNA of the extreme-phenotype bulks and the Xiu94-11 and Zhonghuang47 cultivars were isolated with the Plant Genomic DNA Kit (Tiangen, Beijing, China). The raw read data generated for the Illumina libraries were filtered to produce clean reads, which were then aligned with the Glyma.Wm82.a2.v1 reference genome (http://phytozome.jgi.doe.gov/pz/portal.html) with the genome alignment software BWA [61]. The SNPs and InDels in the two extreme-phenotype bulks and parental cultivars were detected and filtered with the variation analysis software GATK [24,61].

The SNP index of each bulk and the delta SNP index were calculated based on an SNP detected in the two extreme-phenotype bulks during a previous filtering analysis [38]. Three confidence levels $(P<0.1,0.05$, and 0.01) were set for the delta SNP index [38], which was calculated for each SNP position with the following formula:

delta SNP index = SNP index (PRR-resistant bulk) - SNP index (PRR-susceptible bulk).

\subsection{Linkage Analysis and Genetic Mapping of the Candidate Region}

After identifying the RpsX candidate region via QTL-seq, a genetic linkage mapping approach was used to further limit the RpsX candidate region. Publicly available SSR markers in the RpsX candidate region were selected to screen for polymorphisms between the parental cultivars and the genotype mapping population [47]. High-quality InDels identified by whole-genome resequencing were further developed to PCR markers to map the candidate region (Table S4) [24,25]. Combining phenotypic and genotypic results, the genetic linkage analysis of $R p s X$ was completed with the MAPMAKER/EXP (version 3.0) program [62]. The genetic linkage map was constructed with the MapDraw program [63].

\subsection{Phylogenetic Analysis of Soybean Genotypes}

Forty-five soybean genotypes, including landraces/cultivars, were selected to analyze the genetic relationship between Xiu94-11 and Qichadou1/Ludou4. Moreover, in addition to Xiu94-11 and Zhonghuang47, 43 soybean genotypes were subjected to whole-genome resequencing (unpublished data). The DNA of these genotypes was extracted and sent to Annoroad Gene Technology (Beijing, China) for the construction of Illumina sequencing libraries. Raw data were filtered, sequences were aligned with reference genome sequences, and homozygous SNPs were identified and annotated as previously described [24,25]. The homozygous SNPs identified on chromosome 3 for each genotype 
were used to construct a phylogenetic tree according to the neighbor-joining method of the MEGA 6.0 program [64].

\subsection{Analysis of the Allelic Sequences of the Candidate Gene Locus}

The allele sequences of the Glyma.03g027200 locus for the cultivars related to Qichadou 1 were determined by PCR-based Sanger sequencing. The sequencing primers were the same primer pairs used to amplify the RpsQ candidate allele and overlapped new primers designed with the NCBI Primer-BLAST tool (https://www.ncbi.nlm.nih.gov/tools/primer-blast/) (Table S5). A PCR assay was completed with PrimeSTAR ${ }^{\mathrm{TM}}$ HS DNA Polymerase (Takara Biotechnology, Dalian, China). The PCR product obtained for each sample was sequenced by Sangon Biotech (Beijing, China). The resulting sequences were assembled with ContigExpress to obtain the complete Glyma.03g027200 allele sequences. Each sample was amplified and sequenced three times to avoid errors generated during amplification and sequencing.

A multiple sequence alignment involving the obtained sequences was completed with ClustalW, after which a phylogenetic tree was constructed according to the neighbor-joining method (with 1000 bootstrap replicates) of the MEGA 6.0 program [64]. The sequence coding region was confirmed based on the cDNA sequence of the $R p s Q$ allele and the published annotated Williams 82 reference transcripts (https://www.soybase.org/). The coding region sequence was converted to a protein sequence with ExPASy (https://web.expasy.org/translate/). Conserved domains were predicted for the Xiu94-11, Zhonghuang47, Qichadou1, Ludou4, and Williams82 sequences with the Conserved Domain Database (http://www.ncbi.nlm.nih.gov/cdd/) [65] and SMART (http://smart.embl-heidelberg.de/) [66].

\subsection{Validation and Screening of RpsX and Its Alleles in Soybean Genotypes Using the Functional Marker Insert144}

Cultivars containing single $R p s$ genes were genotyped with the Insert144 marker that co-segregated with $R p s Q$. This marker was developed based on the 144-bp insertion in the RpsQ allele. Insert144 was also used to detect the $R p s X$ and $R p s Q / 9$ resistance alleles in 177 soybean cultivars and landraces whose reactions to eight $P$. sojae isolates had been analyzed. The PCR assay was completed as previously described.

Supplementary Materials: Supplementary materials can be found at http://www.mdpi.com/1422-0067/20/8/1809/ s1.

Author Contributions: Z.Z. and S.S. conceived and designed the experiments. C.Z., Y.L., S.S., and C.D. performed the experiments. C.Z. and Y.L. analyzed the data and wrote the manuscript. Z.Z. and S.S. revised the manuscript. All authors read and approved the manuscript.

Funding: This study was supported by the Special Fund for Agroscientific Research in the Public Interest (201303018), the Program of Protection of Crop Germplasm Resources (2017NWB036-12) from the Ministry of Agriculture of the People's Republic of China, the National Infrastructure for Crop Germplasm Resources (NICGR2017-008), and the Scientific Innovation Program of Chinese Academy of Agricultural Sciences.

Acknowledgments: We thank Lijuan Qiu from the Institute of Crop Sciences, Chinese Academy of Agricultural Sciences, and Ran Xu from the Shandong Academy of Agricultural Sciences for supplying the soybean cultivars tested in this study.

Conflicts of Interest: The authors declare that they have no conflict of interest.

\section{Abbreviations}

$\begin{array}{ll}\text { LRR } & \text { Leucine-rich repeat } \\ \text { PRR } & \text { Phytophthora root rot } \\ \text { NGS } & \text { Next-generation sequencing } \\ \text { SNP } & \text { Single nucleotide polymorphism } \\ \text { InDel } & \text { Insertion/deletion } \\ \text { STK } & \text { Serine/threonine protein kinase } \\ \text { NBS } & \text { Nucleotide-binding site }\end{array}$




\section{References}

1. Zhou, Z.; Jiang, Y.; Wang, Z.; Gou, Z.; Lyu, J.; Li, W.; Yu, Y.; Shu, L.; Zhao, Y.; Ma, Y.; et al. Resequencing 302 wild and cultivated accessions identifies genes related to domestication and improvement in soybean. Nat. Biotechnol. 2015, 33, 408-414. [CrossRef] [PubMed]

2. Kaufmann, M.; Gerdemann, J. Root and stem rot of soybean caused by Phytophthora sojae n. sp. Phytopathology 1958, 48, 201-208.

3. Schmitthenner, A.F. Problems and progress in control of Phytophthora root rot of soybean. Plant Dis. 1985, 69, 362-368. [CrossRef]

4. Kamoun, S.; Furzer, O.; Jones, J.D.; Judelson, H.S.; Ali, G.S.; Dalio, R.J.; Roy, S.G.; Schena, L.; Zambounis, A.; Panabières, F.; et al. The Top 10 oomycete pathogens in molecular plant pathology. Mol. Plant Pathol. 2015, 16, 413-434. [CrossRef] [PubMed]

5. Koenning, S.R.; Wrather, J.A. Suppression of soybean yield potential in the continental United States by plant diseases from 2006 to 2009. Plant Health Prog. 2010. Available online: https://www.plantmanagementnetwork. org/pub/php/research/2010/yield/ (accessed on 9 April 2019). [CrossRef]

6. Dorrance, A.E. Management of Phytophthora sojae of soybean: A review and future perspectives. Can. J. Plant Pathol. 2018, 40, 210-219. [CrossRef]

7. Chen, X.; Wang, Y. Phytophthora sojae. In Biological Invasions and Its Management in China; Wan, F., Jiang, M., Zhan, A., Eds.; Springer: Singapore, 2017; pp. 199-223.

8. Tyler, B.M. Phytophthora sojae: Root rot pathogen of soybean and model oomycete. Mol. Plant Pathol. 2007, 8, 1-8. [CrossRef]

9. Sugimoto, T.; Kato, M.; Yoshida, S.; Matsumoto, I.; Kobayashi, T.; Kaga, A.; Hajika, M.; Yamamoto, R.; Watanabe, K.; Aino, M.; et al. Pathogenic diversity of Phytophthora sojae and breeding strategies to develop Phytophthora-resistant soybeans. Breed. Sci. 2012, 61, 511-522. [CrossRef]

10. Tooley, P.W.; Grau, C.R. The relationship between rate-reducing resistance to Phytophthora megasperma f. sp. glycinea and yield of soybean. Phytopathology 1984, 74, 1209-1216. [CrossRef]

11. Dorrance, A.E.; St. McClure, S.A.; Martin, S.K. Effect of partial resistance on Phytophthora stem rot incidence and yield of soybean in Ohio. Plant Dis. 2003, 87, 308-312. [CrossRef]

12. Dorrance, A.E.; Jia, H.; Abney, T.S. Evaluation of soybean differentials for their interaction with Phytophthora sojae. Plant Health Prog. 2004. [CrossRef]

13. Zhang, J.; Xia, C.; Duan, C.; Sun, S.; Wang, X.; Wu, X.; Zhu, Z. Identification and candidate gene analysis of a novel Phytophthora resistance gene Rps10 in a Chinese soybean cultivar. PLoS ONE 2013, 8, e69799. [CrossRef]

14. Zhang, J.; Xia, C.; Wang, X.; Duan, C.; Sun, S.; Wu, X.; Zhu, Z. Genetic characterization and fine mapping of the novel Phytophthora resistance gene in a Chinese soybean cultivar. Theor. Appl. Genet. 2013, 126, 1555-1561. [CrossRef] [PubMed]

15. Lin, F.; Zhao, M.; Ping, J.; Johnson, A.; Zhang, B.; Abney, T.S.; Hughes, T.; Ma, J. Molecular mapping of two genes conferring resistance to Phytophthora sojae in a soybean landrace PI 567139B. Theor. Appl. Genet. 2013, 126, 2177-2185. [CrossRef] [PubMed]

16. Sun, J.; Li, L.; Zhao, J.; Huang, J.; Yan, Q.; Xing, H.; Guo, N. Genetic analysis and fine mapping of RpsJS, a novel resistance gene to Phytophthora sojae in soybean [Glycine max (L.) Merr.]. Theor. Appl. Genet. 2014, 127, 913-919. [CrossRef] [PubMed]

17. Ping, J.; Fitzgerald, C.; Zhang, C.; Lin, F.; Bai, Y.; Wang, D.; Aggarwal, R.; Rehman, M.; Crasta, O.; Ma, J. Identification and molecular mapping of Rps11, a novel gene conferring resistance to Phytophthora sojae in soybean. Theor. Appl. Genet. 2016, 129, 445-451. [CrossRef] [PubMed]

18. Li, L.; Lin, F.; Wang, W.; Ping, J.; Fitzgerald, J.; Zhao, M.; Li, S.; Sun, L.; Cai, C.; Ma, J. Fine mapping and candidate gene analysis of two loci conferring resistance to Phytophthora sojae in soybean. Theor. Appl. Genet. 2016, 129, 2379-2386. [CrossRef]

19. Cheng, Y.; Ma, Q.; Ren, H.; Xia, Q.; Song, E.; Tan, Z.; Li, S.; Zhang, G.; Nian, H. Fine mapping of a Phytophthora-resistance gene RpsWY in soybean (Glycine max L.) by high-throughput genome-wide sequencing. Theor. Appl. Genet. 2017, 130, 1041-1051. [CrossRef] [PubMed] 
20. Li, Y.; Sun, S.; Zhong, C.; Wang, X.; Wu, X.; Zhu, Z. Genetic mapping and development of co-segregating markers of $R p s Q$, which provides resistance to in soybean. Theor. Appl. Genet. 2017, 130, 1223-1233. [CrossRef]

21. Niu, J.; Guo, N.; Sun, J.; Li, L.; Cao, Y.; Li, S.; Huang, J.; Zhao, J.; Zhao, T.; Xing, H. Fine mapping of a resistance gene RpsHN that controls Phytophthora sojae using recombinant inbred lines and secondary populations. Front. Plant Sci. 2017, 8, 538. [CrossRef]

22. Sahoo, D.K.; Abeysekara, N.S.; Cianzio, S.R.; Robertson, A.E.; Bhattacharyya, M.K. A Novel Phytophthora sojae Resistance Rps12 Gene Mapped to a Genomic Region That Contains Several Rps Genes. PLoS ONE 2017, 12, e0169950. [CrossRef] [PubMed]

23. Qin, J.; Song, Q.; Shi, A.; Li, S.; Zhang, M.; Zhang, B. Genome-wide association mapping of resistance to Phytophthora sojae in a soybean [Glycine max (L.) Merr.] germplasm panel from maturity groups IV and V. PLoS ONE 2017, 12, e0184613. [CrossRef] [PubMed]

24. Zhong, C.; Sun, S.; Li, Y.; Duan, C.; Zhu, Z. Next-generation sequencing to identify candidate genes and develop diagnostic markers for a novel Phytophthora resistance gene, RpsHC18, in soybean. Theor. Appl. Genet. 2017, 131, 525-538. [CrossRef]

25. Zhong, C.; Sun, S.; Yao, L.; Ding, J.; Duan, C.; Zhu, Z. Fine mapping and identification of a novel phytophthora root rot resistance locus RpsZS18 on Chromosome 2 in Soybean. Front. Plant Sci. 2018, 9, 44. [CrossRef]

26. Tian, M.; Zhao, L.; Li, S.; Huang, J.; Sui, Z.; Wen, J.; Li, Y. Pathotypes and metalaxyl sensitivity of Phytophthora sojae and their distribution in Heilongjiang, China 2011-2015. J. Gen. Plant Pathol. 2016, 82, 132-141. [CrossRef]

27. Wu, M.; Li, B.; Liu, P.; Weng, Q.; Zhan, J.; Chen, Q. Genetic analysis of Phytophthora sojae populations in Fujian, China. Plant Pathol. 2017, 66, 1182-1190. [CrossRef]

28. Dorrance, A.E.; Kurle, J.; Robertson, A.E.; Bradley, C.A.; Giesler, L.; Wise, K.; Concibido, V.C. Pathotype diversity of Phytophthora sojae in eleven states in the United States. Plant Dis. 2016, 100, 1429-1437. [CrossRef]

29. Schmitthenner, A.F. Phytophthora rot of soybean. In Compendium of Soybean Diseases, 4 th ed.; The American Phytopathological Society Press: St. Paul, MN, USA, 1999; pp. 39-42.

30. Costamilan, L.M.; Clebsch, C.C.; Soares, R.M.; Seixas, C.D.S.; Godoy, C.V.; Dorrance, A.E. Pathogenic diversity of Phytophthora sojae pathotypes from Brazil. Eur. J. Plant Pathol. 2013, 135, 845-853. [CrossRef]

31. Dorrance, A.E.; Schmitthenner, A.F. New sources of resistance to Phytophthora sojae in the soybean plant introductions. Plant Dis. 2000, 84, 1303-1308. [CrossRef]

32. Zhang, J.; Sun, S.; Wang, G.; Duan, C.; Wang, X.; Wu, X.; Zhu, Z. Characterization of Phytophthora resistance in soybean cultivars/lines bred in Henan province. Euphytica 2014, 196, 375-384. [CrossRef]

33. Scheben, A.; Batley, J.; Edwards, D. Genotyping by sequencing approaches to characterise crop genomes: Choosing the right tool for the right application. Plant Biotechnol. J. 2016, 15, 149-161. [CrossRef]

34. Wallace, J.G.; Mitchell, S.E. Genotyping-by-sequencing. Curr. Protoc. Plant Biol. 2017, 2, 64-77.

35. Grant, D.; Nelson, R.T.; Cannon, S.B.; Shoemaker, R.C. SoyBase, the USDA-ARS soybean genetics and genomics database. Nucleic Acids Res. 2009, 38, 843-846. [CrossRef]

36. Schmutz, J.; Cannon, S.B.; Schlueter, J.; Ma, J.; Mitros, T.; Nelson, W.; Hyten, D.L.; Song, Q.; Thelen, J.J.; Cheng, J.; et al. Genome sequence of the palaeopolyploid soybean. Nature 2010, 463, 178-183. [CrossRef]

37. Song, Q.; Jenkins, J.; Jia, G.; Hyten, D.L.; Pantalone, V.; Jackson, S.A.; Schmutz, J.; Cregan, P.B. Construction of high resolution genetic linkage maps to improve the soybean genome sequence assembly Glyma1. 01. BMC Genom. 2016, 17, 33. [CrossRef]

38. Takagi, H.; Abe, A.; Yoshida, K.; Kosugi, S.; Natsume, S.; Mitsuoka, C.; Uemura, A.; Utsushi, H.; Tamiru, M.; Takuno, S.; et al. QTL-seq: Rapid mapping of quantitative trait loci in rice by whole genome resequencing of DNA from two bulked populations. Plant J. 2013, 74, 174-183. [CrossRef]

39. Lu, H.; Lin, T.; Klein, J.; Wang, S.; Qi, J.; Zhou, Q.; Sun, J.; Zhang, Z.; Weng, Y.; Huang, S. QTL-seq identifies an early flowering QTL located near flowering locus T in cucumber. Theor. Appl. Genet. 2014, 127, 1491-1499. [CrossRef]

40. Illa-Berenguer, E.; Van Houten, J.; Huang, Z.; van der Knaap, E. Rapid and reliable identification of tomato fruit weight and locule number loci byQTL-seq. Theor. Appl. Genet. 2015, 128, 1329-1342. [CrossRef]

41. Das, S.; Upadhyaya, H.D.; Bajaj, D.; Kujur, A.; Badoni, S.; Laxmi; Kumar, V.; Tripathi, S.; Gowda, C.L.; Sharma, S.; et al. Deploying QTL-seq for rapid delineation of a potential candidate gene underlying major trait-associated QTL in chickpea. DNA Res. 2015, 22, 193-203. [CrossRef] 
42. Singh, V.K.; Khan, A.W.; Jaganathan, D.; Thudi, M.; Roorkiwal, M.; Takagi, H.; Garg, V.; Kumar, V.; Chitikineni, A.; Gaur, P.M.; et al. QTL-seq for rapid identification of candidate genesfor 100-seed weight and root/total plant dry weight ratio under rained conditions in chickpea. Plant Biotechnol. J. 2016, 14, 2110-2119. [CrossRef]

43. Singh, V.K.; Khan, A.W.; Saxena, R.K.; Kumar, V.; Kale, S.M.; Sinha, P.; Chitikineni, A.; Pazhamala, L.T.; Garg, V.; Sharma, M.; et al. Next-generation sequencing for identification of candidate genes for Fusarium wilt and sterility mosaic disease in pigeonpea (Cajanus cajan). Plant Biotechnol. J. 2016, 14, 1183-1194. [CrossRef]

44. Singh, V.K.; Khan, A.W.; Saxena, R.K.; Sinha, P.; Kale, S.M.; Parupalli, S.; Kumar, V.; Chitikineni, A.; Vechalapu, S.; Sameer Kumar, C.V.; et al. Indel-seq: A fast-forward genetics approach for identification of trait-associated putative candidate genomic regions and its application in pigeonpea (Cajanus cajan). Plant Biotechnol. J. 2017, 15, 906-914. [CrossRef]

45. Pandey, M.K.; Khan, A.W.; Singh, V.K.; Vishwakarma, M.K.; Shasidhar, Y.; Kumar, V.; Garg, V.; Bhat, R.S.; Chitikineni, A.; Janila, P.; et al. QTL-seq approach identified genomic regions and diagnostic markers for rust and late leaf spot resistance in groundnut (Arachis hypogaea L.). Plant Biotechnol. J. 2017, 15, 927-941. [CrossRef]

46. Lin, M.S.; Lin, S.F. Pedigree analysis of soybean varieties. Bot. Bull. Acad. Sin. 1994, 35, 87-93.

47. Gao, H.; Bhattacharyya, M.K. The soybean-Phytophthora resistance locus Rps1-k encompasses coiled coil-nucleotide binding leucine rich repeat-like genes and repetitive sequences. BMC Plant Biol. 2008, 8, 29. [CrossRef]

48. Gao, H.; Narayanan, N.N.; Ellison, L.; Bhattacharyya, M.K. Two classes of highly similar coiled coil-nucleotide binding-leucine rich repeat genes isolated from the Rps1- $k$ locus encode Phytophthora resistance in soybean. Mol. Plant Microb. Interact. 2005, 18, 1035-1045. [CrossRef]

49. Feuillet, C.; Schachermayr, G.; Keller, B. Molecular cloning of a new receptor-like kinase gene encoded at the Lrl0 disease resistance locus of wheat. Plant J. 1997, 11, 45-52. [CrossRef]

50. Song, W.Y.; Wang, G.L.; Chen, L.L.; Kim, H.S.; Pi, L.Y.; Holsten, T.; Gardner, J.; Wang, B.; Zhai, W.X.; Zhu, L.H.; et al. A receptor kinase-like protein encoded by the rice disease resistance gene, Xa21. Science 1995, 27, 1804-1806. [CrossRef]

51. Wang, G.L.; Ruan, D.L.; Song, W.Y.; Sideris, S.; Chen, L.; Pi, L.Y.; Zhang, S.; Zhang, Z.; Fauquet, C.; Gautd, B.S.; et al. Xa21D encodes a receptor-like molecule with a leucine-rich repeat domain that determines race-specific recognition and is subject to adaptive evolution. Plant Cell 1998, 10, 765-779. [CrossRef]

52. Sun, X.; Cao, Y.; Yang, Z.; Xu, C.; Li, X.; Wang, S.; Zhang, Q. Xa26, a gene conferring resistance to Xanthomonas oryzae pv. oryzae in rice, encodes an LRR receptor kinase-like protein. Plant J. 2004, 37, 517-527. [CrossRef]

53. Padmarasu, S.; Sargent, D.J.; Patocchi, A.; Troggio, M.; Baldi, P.; Linsmith, G.; Poles, L.; Jänsch, M.; Kellerhals, M.; Tartarini, S.; et al. Identification of a leucine-rich repeat receptor-like serine/threonine-protein kinase as a candidate gene for Rvi12 (Vb)-based apple scab resistance. Mol. Breed. 2018, 38, 73. [CrossRef]

54. Dodds, P.N.; Lawrence, G.J.; Ellis, J.G. Six amino acidchanges confined to the leucine-rich repeat beta-strand/beta-turnmotif determine the difference between the P and P2 rust resistance specificities in flax. Plant Cell 2001, 13, 163-178.

55. Jones, D.; Jones, J.D. The role of leucine-rich repeat proteins in plant defenses. Adv. Bot. Res. 1997, $24,89-167$.

56. Ellis, J.; Dodds, P.; Pryor, T. The generation of plant disease resistance gene specificities. Trends Plant Sci. 2000, 5, 373-379. [CrossRef]

57. Ramkumar, G.; Madhav, M.S.; Devi, S.R.; Umakanth, B.; Pandey, M.K.; Prasad, M.S.; Sundaram, R.M.; Viraktamath, B.C.; Babu, V.R. Identification and validation of novel alleles of rice blast resistant gene Pi54, and analysis of their nucleotide diversity in landraces and wild Oryza species. Euphytica 2016, 209, 725-737. [CrossRef]

58. Thakur, S.; Singh, P.K.; Das, A.; Rathour, R.; Variar, M.; Prashanthi, S.K.; Singh, A.K.; Singh, U.D.; Chand, D.; Singh, N.K.; et al. Extensive sequence variation in rice blast resistance gene Pi54 makes it broad spectrum in nature. Front. Plant Sci. 2015, 6, 345. [CrossRef]

59. Wicker, T.; Yahiaoui, N.; Keller, B. Illegitimate recombination is a major evolutionary mechanism for initiating size variation in plant resistance genes. Plant J. 2007, 51, 631-641. [CrossRef]

60. Lawrence, G.J.; Anderson, P.A.; Dodds, P.N.; Ellis, J.G. Relationships between rust resistance genes at the M locus in flax. Mol. Plant Pathol. 2010, 11, 19-32. [CrossRef] 
61. McKenna, A.; Hanna, M.; Banks, E.; Sivachenko, A.; Cibulskis, K.; Kernytsky, A.; DePristo, M.A. The Genome Analysis Toolkit: A MapReduce framework for analyzing next-generation DNA sequencing data. Genome Res. 2010, 20, 1297-1303. [CrossRef]

62. Lincoln, S.E.; Daly, M.J.; Lander, E.S. Constructing Genetic Linkage Maps with MAPMAKER/EXP Version 3.0: A Tutorial and Reference Manual. A Whitehead Institute for Biomedical Research Technical Report. 1993, Volume 3. Available online: http://home.cc.umanitoba.ca/ \{\}psgendb/birchhomedir/doc/mapmaker/ mapmaker.tutorial.pdf (accessed on 9 April 2019).

63. Liu, R.H.; Meng, J.L. MapDraw: A microsoft excel macro for drawing genetic linkage maps based on given genetic linkage data. Hereditas 2003, 25, 317-321.

64. Tamura, K.; Stecher, G.; Peterson, D.; Filipski, A.; Kumar, S. MEGA6: Molecular evolutionary genetics analysis version 6.0. Mol. Biol. Evol. 2013, 30, 2725-2729. [CrossRef]

65. Marchler-Bauer, A.; Derbyshire, M.K.; Gonzales, N.R.; Lu, S.; Chitsaz, F.; Geer, L.Y.; Geer, R.C.; He, J.; Gwadz, M.; Hurwitz, D.I.; et al. CDD: NCBI's conserved domain database. Nucleic Acids Res. 2015, 43, 222-226. [CrossRef]

66. Letunic, I.; Doerks, T.; Bork, P. SMART: Recent updates, new developments and status in 2015. Nucleic Acids Res. 2015, 43, 257-260. [CrossRef]

(C) 2019 by the authors. Licensee MDPI, Basel, Switzerland. This article is an open access article distributed under the terms and conditions of the Creative Commons Attribution (CC BY) license (http://creativecommons.org/licenses/by/4.0/). 